Research Article

\title{
Protective Effect of the MCP-1 Gene Haplotype against Schizophrenia
}

\author{
Hana Saoud $\mathbb{D},{ }^{1}$ Oumaima Inoubli $\left(\mathbb{D},{ }^{1}\right.$ Sihem Ben Fredj $\mathbb{D},{ }^{2}$ Mohsen Hassine, ${ }^{3}$ \\ Bochra Ben Mohamed, ${ }^{4}$ Lotfi Gaha, ${ }^{4}$ and Besma Bel Hadj Jrad ${ }^{1}$ \\ ${ }^{1}$ Laboratory of Genetics, Biodiversity and Bioresource Valorization, Higher Institute of Biotechnology of Monastir, \\ University of Monastir, Monastir, Tunisia \\ ${ }^{2}$ Department of Epidemiology, Farhat Hached University Hospital, Sousse, Tunisia \\ ${ }^{3}$ Hematology Department, Fattouma Bourguiba University Hospital, TN 5000, Monastir, Tunisia \\ ${ }^{4}$ Department of Psychiatry and Vulnerability to Psychoses Laboratory-CHU Monastir, University of Monastir, Monastir, Tunisia
}

Correspondence should be addressed to Hana Saoud; hana.seoud@yahoo.fr

Received 13 August 2019; Revised 28 October 2019; Accepted 15 November 2019; Published 2 December 2019

Academic Editor: Milton O. Moraes

Copyright (c) 2019 Hana Saoud et al. This is an open access article distributed under the Creative Commons Attribution License, which permits unrestricted use, distribution, and reproduction in any medium, provided the original work is properly cited.

\begin{abstract}
While cytokines and their genetic variants have been intensively studied in schizophrenia, little attention has been focused on chemokines in the last years. The monocyte chemoattractant protein 1 (MCP-1) is known to attract peripheral monocytes to the brain during an inflammatory reaction and to affect the T helper (Th) cell development by stimulating Th2 polarization. Owing to the neuroinflammation in schizophrenia and the variable level of MCP-1 in these patients' sera, we proposed to analyze the impact of functional genetic variants of the MCP-1 gene (MCP-1-2518A/G (rs1024611), MCP-1-362G/C (rs2857656), and MCP-1 int1del554-567 (rs3917887)) in schizophrenic patients. We conducted a case-control study on a Tunisian population composed of 200 patients and 200 controls using RFLP-PCR. Our results indicated that the minor alleles (-2518G and Del554-567) were significantly more prevalent in controls than in patients $(P=0.001$ /adjusted $\mathrm{OR}=0.42, P=0.04$ /adjusted $\mathrm{OR}=0.64)$, whereas, for $-362 \mathrm{C}$ minor allele, increased risk of schizophrenia was revealed $(P=0.001$, adjusted $\mathrm{OR}=2.38)$. In conclusion, we have identified the haplotype combination -2581G/-362G/int1del554-567 that could mediate protection against schizophrenia $(P=0.0038$, $\mathrm{OR}=0.19)$ and the effect could result more strongly from the MCP-1 $-2582 \mathrm{G}$ with $-362 \mathrm{G}$ variants, whereas the effect of int1del554567 may in part be explained by its LD with -362 .
\end{abstract}

\section{Introduction}

Schizophrenia (SCZ) is a chronic and disabling neuropsychiatric disease. It usually appears in the late second and third decades of life with a varied symptomatology, generally belonging to three classes, namely, positive symptoms, which include hallucinations, delusions, and thought disorganization; negative symptoms, which refer to reduced motivation and emotional vibrancy; and cognitive deficits that reflect in particular an alteration in working memory, executive functions, and attention processes [1]. The causes remain unclear, and given the heterogeneous nature of the disorder, it is likely that there are multiple contributing etiological agents. Several studies have supported the role of an inflammatory component. In line with it, increased levels of cytokines such as TNF- $\alpha$, IL- 6 , IFN- $\gamma$, and IL- $1 \beta$ were detected in the blood and cerebrospinal fluid in the first onset and acute relapse patients with schizophrenia [2]. A postmortem study reported that the expression of a microglial gene is dysregulated [3] and aberrant functions of microglia have potential implications in this disorder $[4,5]$. However, recent knowledge of the high degree of macrophage plasticity and microglia has brought to light that, in inflammatory conditions, microglial cells polarize to M1 phenotype and produce proinflammatory cytokines/mediators including IL- $1 \beta$, IL- 6 , TNF- $\alpha$, CCL2, ROS, and NO $[6,7]$. This prompted a renewed interest in the participation of chemokines which are a family of small cytokines whose 
name is derived from their ability to induce directed chemotaxis in nearby responsive cells. In particular, analyses revealed an increased number of monocytes and elevated levels of CCL2 in the first-episode psychosis (FEP) patients and multiple-episode schizophrenia (MES) individuals $[8,9]$, suggesting that this chemokine may contribute to the development of this disorder.

Monocyte chemoattractant protein-1 (MCP-1), known as CCL2 (chemokine (C-C motif) ligand 2), is a small cytokine that belongs to the CC chemokine family. It is not only expressed in neuroinflammatory conditions but is also constitutively present in the brain in both glial cells [10-13] and neuron [14]. In addition to being considered as a potent mediator for the attraction of monocytes and macrophages to the sites of inflammation, MCP-1 mediates the transendothelial migration of inflammatory cells across the blood-brain barrier and modulates the local inflammatory response by forming chemotactic gradients within the CNS [15]. Variation in MCP-1 expression can be influenced by polymorphic variants of the gene, which is mapped to chromosome 17 (17q11.2-q12) [16]. Among various polymorphisms, two functional genetic variations within the MCP-1 gene promoter, rs1024611 A/G and rs2857656 G/C, were linked to variable levels of CCL2 $[17,18]$.

The $-2518 \mathrm{~A} / \mathrm{G}$ (rs1024611) polymorphism is located in the distal regulatory region of the MCP-1 gene relative to the major transcriptional start site of the gene that can influence the transcriptional activity of MCP-1 and contributes to susceptibility to multiple sclerosis, Alzheimer's disease, and major depressive disorder [18-21]. The second polymorphism, MCP-1 rs2857656 (-362 G/C), has been identified in the proximal promoter region of the gene and was reported to increase the risk of spinal tuberculosis and carotid atherosclerosis by enhancing MCP-1 expression levels $[17,22]$. Also, a 14 base-pair deletion in the first MCP-1 intron, int1del554-567 (rs3917887), which could affect the transcriptional activity of the gene has been analyzed [23]. Considering the role of MCP-1 in modulating immune system response together with the relationship between immune abnormalities and schizophrenia, the primary aim of this study was to investigate the potential role of these three single-nucleotide polymorphisms in conferring susceptibility to or protection against schizophrenia.

\section{Material and Methods}

2.1. Study Participants. The study included 200 patients hospitalized or received in out-patient services at the Psychiatry Department of Monastir Hospital, Tunisia. All of them were diagnosed according to DSM-IV (Diagnostic and Statistical Manual of Mental Disorders, the fourth version) criteria for schizophrenia [24]. The selection was based on the exclusion of schizoaffective disorder and other mental pathologies and severe physical diseases such as cancer. For the patient to be treated and to be considered as true schizophrenic, continuous signs of the disturbance must last longer than 6 months. This $6-$ month period must comprise at the minimum one month of active phase symptoms. Total scores and subscores for positive, negative, and general symptoms were collected. In the current work, $45.9 \%$ of patients met the diagnostic criteria for undifferentiated, $32 \%$ for paranoid, and $19.8 \%$ for disorganized subtypes of schizophrenia. The average age of the cohort was $38.71 \pm 10.69$ years (range $18-65$ years) with $19.5 \%$ females and $80.5 \%$ males (sex ratio $=4.12$ ) and the onset age varied from 10 to 47 years. We used the definition of Meltzer et al. [25], which equates the age at onset of schizophrenia with the first occurrence of positive psychotic symptoms (mean age of onset \pm SD $25.06 \pm 7.59$ years).

The control group consisted of 200 healthy volunteers with a mean age of $32.71 \pm 10.8$ years, ranging from 18 to 65 years and a sex ratio of 3.52 (19.9\% females and $70.1 \%$ males). They were recruited among the blood donors of the same Monastir Hospital, Tunisia. The main inclusion criteria for controls were as follows: being free of any psychiatric illness, nonsubstance abuse, and without a family history of psychiatric disorders.

The institutional ethics committee of the Higher Institute of Biotechnology of Monastir in Tunisia approved the research protocol, and written consent was provided by each participant or a family member before blood collection.

2.2. DNA Extraction and Genotyping. Genomic DNA was extracted from the whole EDTA-anticoagulated blood, according to the salting-out technique [26]. Genotyping was determined by the restriction fragment length polymorphism analysis of PCR-amplified product (RFLP-PCR). All primers were designed using genomic sequences obtained from the National Center for Biotechnology Information (http://www. ncbi.nlm.nih.gov/) and Primer-BLAST.

Details including primers and restriction enzyme with product sizes are listed in Table 1.

2.3. Statistical Analysis. For genetic association analysis, each polymorphism was tested for deviations from Hardy-Weinberg equilibrium in patient and healthy groups. Statistical analysis was performed using the SPSS software (version 23, Armonk, NY, USA). The distribution of genotypes or alleles of chemokine genes between cases and controls was compared by the Chi-square test (or Fisher's exact test when $n<5$ ). Odds ratios (OR) and 95\% confidence intervals (CI) were also calculated whenever $\chi^{2}$ test was significant. Differences between groups were deemed to be significant at $P<0.05$ for all tests. To calculate the linkage disequilibrium (LD) expressed as $D^{\prime}$, the Haploview version 4.2 was used and the graphical view was generated [27]. For haplotype reconstruction, the SNPStats online software (https://www.snpstats.net/start.htm) was performed.

A binary logistic regression model with categorical and quantitative independent variables was done, adjusting for the effects of age and gender through the analysis of deviance from a sequential addition of each variable.

\section{Results}

The determination of genotypic and allelic frequencies of investigating SNPs is given in Table 2. Genotype distribution 
TABLE 1: Primers, fragment patterns, and enzymes used for RFLP analysis.

\begin{tabular}{|c|c|c|c|}
\hline Polymorphisms & Primers $\left(5^{\prime}-3^{\prime}\right)$ & Amplicon sizes (bp) & Restriction enzymes \\
\hline$-2518 \mathrm{~A} / \mathrm{G}(\mathrm{rs} 1024611)$ & $\begin{array}{c}\text { F: GCTCCGGGCCCAGTATCT } \\
\text { R: ACAGGGAAGGTGAAGGGTATGA }\end{array}$ & $\begin{array}{c}182,54 \\
236\end{array}$ & PvuII \\
\hline 362C/G (rs2857656) & $\begin{array}{l}\text { F: CTAGGCTTCTATGATGCTAC } \\
\text { R: TCCATTCACTGCTGAGACCA }\end{array}$ & $\begin{array}{c}201,110 \\
311\end{array}$ & Hрy188I \\
\hline CCL2I/D (rs3917887) & $\begin{array}{c}\text { F: GCTGATCTTCCCTGGTGCTGAT } \\
\text { R: CATTAAATCCCAGTGCTTCTGCCTA }\end{array}$ & $\begin{array}{l}\mathrm{I}: 202 \\
\mathrm{D}: 188\end{array}$ & - \\
\hline
\end{tabular}

F: forward primer; R: reverse primer; I: insertion; D: deletion. In order to ratify the data generated by the PCR-RFPL method, we analyzed $25 \%$ of the samples randomly.

TABLE 2: Genotype distribution and allele frequencies of MCP-1 polymorphisms in healthy controls and patients with Schizophrenia.

\begin{tabular}{|c|c|c|c|c|c|}
\hline Genotype & Healthy controls $n=200(\%)$ & Patients $n=200(\%)$ & OR $(95 \% \mathrm{CI})$ & $P$ value & AIC \\
\hline \multicolumn{6}{|l|}{ MCP-1 -2518A/G (rs1024611) } \\
\hline \multicolumn{6}{|l|}{ Genotypes } \\
\hline $\mathrm{AA}$ & $124(62 \%)$ & $166(83 \%)$ & & & \\
\hline AG & $69(34.5 \%)$ & $33(16.5 \%)$ & $0.35(0.22-0.57)$ & $0.000015^{*}$ & \\
\hline GG & 7 (3.5\%) & $1(0.5 \%)$ & $0.10(0.004-0.7)$ & $0.015^{*}$ & 536.4 \\
\hline Dominant (AA vs $A G+G G)$ & & & $0.33(0.20-0.53)$ & $0.0000023^{*}$ & $536^{*}$ \\
\hline Recessive (AA + AG vs GG) & & & $0.13(0.006-0.9)$ & 0.067 & 553.4 \\
\hline $\mathrm{HWE}^{\mathrm{a}}$ & 0.48 & 0.22 & & & \\
\hline \multicolumn{6}{|l|}{ Alleles } \\
\hline A & $317(79.25 \%)$ & $365(91.25 \%)$ & & & \\
\hline $\mathrm{G}$ & $83(20.75 \%)$ & $35(8.75 \%)$ & $0.36(0.23-0.55)$ & $0.000001^{*}$ & \\
\hline \multicolumn{6}{|l|}{ MCP-1 -362 G/C (rs2857656) } \\
\hline \multicolumn{6}{|l|}{ Genotypes } \\
\hline GG & $67(33.5 \%)$ & $46(23 \%)$ & & & \\
\hline GC & $92(46 \%)$ & $88(44 \%)$ & $1.3(0.86-2.24)$ & 0.17 & \\
\hline $\mathrm{CC}$ & $41(20.5 \%)$ & $66(33 \%)$ & $2.33(1.36-4.03)$ & $0.001^{*}$ & 552.4 \\
\hline Dominant (GG vs GC + CC) & & & $1.68(1.08-2.63)$ & $0.02^{*}$ & 554.8 \\
\hline Recessive (GG + GC vs CC) & & & $1.9(1.21-3.01)$ & $0.004^{*}$ & $552^{*}$ \\
\hline $\mathrm{HWE}^{\mathrm{a}}$ & 0.82 & 2.46 & & & \\
\hline \multicolumn{6}{|l|}{ Alleles } \\
\hline G & $226(56.5 \%)$ & $180(45 \%)$ & & & \\
\hline $\mathrm{C}$ & $174(43.5 \%)$ & $220(55 \%)$ & $1.58(1.2-2.09)$ & $0.001^{*}$ & \\
\hline \multicolumn{6}{|l|}{ MCP-1 int1del554-567 (rs3917887) } \\
\hline \multicolumn{6}{|l|}{ Genotypes } \\
\hline Ins/Ins & $136(68 \%)$ & $154(77 \%)$ & & & \\
\hline Ins/Del & $59(29.5 \%)$ & $41(20.5 \%)$ & $0.61(0.38-0.97)$ & $0.03^{*}$ & \\
\hline Del/Del & $5(2.5 \%)$ & $5(2.5 \%)$ & $0.88(0.23-3.3)$ & 0.8 & 553.1 \\
\hline Dominant (Ins/Ins vs Ins/Del + Del/Del) & - & - & $0.63(0.4-0.98)$ & $0.04^{*}$ & $551.4^{*}$ \\
\hline Recessive (Ins/Ins + Ins/Del vs Del/Del) & - & - & $1(0.26-3.77)$ & $>0.99$ & 555.7 \\
\hline $\mathrm{HWE}^{\mathrm{a}}$ & 0.22 & 1.23 & & & \\
\hline \multicolumn{6}{|l|}{ Alleles } \\
\hline Ins & $331(82.75 \%)$ & 349 (87.25\%) & & & \\
\hline Del & $69(17.25 \%)$ & $51(12.75 \%)$ & $0.7(0.47-1.03)$ & 0.075 & \\
\hline
\end{tabular}

OR: odds ratio; CI: confidence interval; HC: healthy controls; ${ }^{*} P<0.05$; HWE: Hardy-Weinberg equilibrium; ${ }^{\mathrm{a} C h i-s q u a r e}$ value AIC (Akaike information criterion) provides a means for model selection.

was in accordance with HWE in both controls and cases for all markers.

The promoter allele $-2581 \mathrm{G}$ was significantly associated with resistance to schizophrenia $(P=0.000001$; $\mathrm{OR}=0.36)$, whereas, for $-362 \mathrm{C}$ allele, an increased risk of schizophrenia was revealed $(P=0.001$; $\mathrm{OR}=1.58)$. There was no statistically significant difference between the patient and the control groups in terms of allele frequency of the MCP-1 int1del554-567 polymorphism $(P=0.075$;
$\mathrm{OR}=0.7)$. A strong association was seen for both heterozygous and homozygous carriers of MCP-1 -2518G (OR $=0.35, \quad P=0.000015 ; \quad \mathrm{OR}=0.1, \quad P=0.015$, respectively). Differently, only the frequency of MCP-1 -362 (CC) minor homozygote genotype was shown to be significantly more prevalent among healthy controls $(\mathrm{OR}=2.33, P=0.001)$. Yet, the CCL2I/D heterozygous genotype frequency was found to be higher in patients $(\mathrm{OR}=0.61, P=0.03)$. 
These associations remain the most highly significant with the lowest Akaike information criterion (AIC) according to the dominant model of inheritance for $-2518 \mathrm{~A} / \mathrm{G}$ and int1del554-567 polymorphisms and to the recessive model of inheritance for the $-362 \mathrm{G} / \mathrm{C}$ polymorphism (Table 2).

As a way to boost statistical power, phenotypic parameters were correlated with the genetic data. Thus, we classified schizophrenic patients into cases with and without specific characteristics to give a robust reduction in the heterogeneity of this pathology. When we stratified the studied populations, according to their gender, our results showed that the $-2518 \mathrm{G}$ allele frequency was higher in male controls $(\mathrm{OR}=0.43, P=0.001)$ but the $-362 \mathrm{C}$ allele frequency was increased among male patients $(\mathrm{OR}=1.79$, $P=0.0004)$. Similar results have been found for females without reaching the statistical significance, potentially related to the small size of the female population analyzed (OR=0.7, $\quad P=0.13 ; \quad \mathrm{OR}=1.13, \quad P=0.7, \quad$ respectively). However, for the int1del554-567 Del, allele differences were not significant for both sexes (Males: $\mathrm{OR}=0.7, P=0.13$, Females: $\mathrm{OR}=0.44, P=0.06$ ).

Furthermore, when patients were stratified on the basis of clinical subtypes, genotype and allele frequencies of the SNP at position-2518 were higher in healthy individuals regardless of the three schizophrenia forms. Concerning the $-362 \mathrm{G} / \mathrm{C}$ polymorphism, the association remained significant with paranoid and undifferentiated schizophrenia but not with the disorganized type of the disease. The genetic distribution of the MCP-1 int1del554-567 polymorphism between the studied groups did not reveal any association with the different clinical forms. To control the possible bias of potentially confusing confounders, a logistic regression model including sex, age, and genotype variables, conforming to the appropriate genetic model of inheritance, showed that the mutated genotypes MCP-1 -2518 (AG + GG), MCP-1 -362 (CC), and MCP-1 int1del554-567 $($ Ins/Del + Del/Del) were correlated with schizophrenia ( $P=0.001 /$ adjusted $\quad \mathrm{OR}=0.42, \quad P=0.001 /$ adjusted $\mathrm{OR}=2.38$, and $P=0.04$ /adjusted $\mathrm{OR}=0.6$, respectively) and the same result was confirmed for the association with the different clinical forms.

Haploview analysis revealed strong LD between rs2857656 and rs3917887 $\left(D^{\prime}=77\right)$ as compared to that conferred by rs1024611 with $\operatorname{rs3917887}\left(D^{\prime}=27\right)$ and rs2857656 $\left(D^{\prime}=10\right)$ (Figure 1).

Only haplotypes having a frequency of more than 5\% were considered. Statistically significant differences in frequencies between cases and controls were observed in one out of the four combinations present (G-G-Ins) containing the mutated $\mathrm{G}$ allele of the rs102411. The G-G-Ins haplotype was associated with protection against schizophrenia as it was higher among healthy subjects compared to patients $(12.96 \%$ vs $2.48 \%)$. Estimated haplotypes constructed for the three markers in both patients and controls are presented in Table 3.

\section{Discussion}

Dysregulations of the immune system are being increasingly studied and seem to play a major role in the pathophysiology

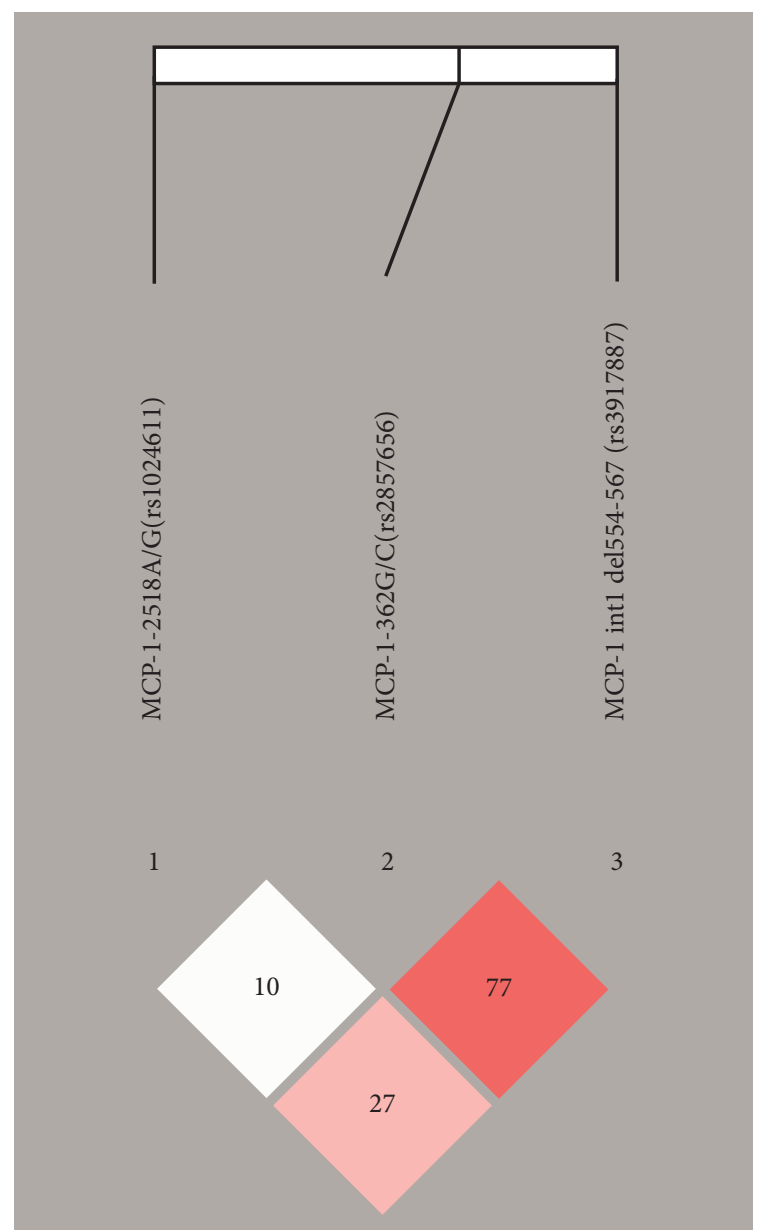

FIGURE 1: Linkage disequilibrium (LD) map of the three MCP-1 SNPs genotyped using Haploview. The positions of the tested variants are indicated above the Haploview output. The LD between specific pairs of MCP-1 SNPs is displayed by the color scheme, which represents LD relationships, based on $D^{\prime}$ values (linkage coefficient according to Lewontin) multiplied by 100 . Bright red squares illustrate high $\mathrm{LD}$, squares in shades of pink/red reveal moderate $\mathrm{LD}$, and white squares indicate no statistically significant difference of LD.

TABLE 3: Haplotype analysis of MCP-1 polymorphisms in patients with schizophrenia and control subjects.

\begin{tabular}{|c|c|c|c|c|}
\hline \multirow[t]{2}{*}{ CCL2 haplotypes } & \multicolumn{2}{|c|}{$\begin{array}{c}\text { Percent } \\
\text { haplotype } \\
\text { frequencies }\end{array}$} & \multirow[t]{2}{*}{ OR $(95 \% \mathrm{CI})$} & \multirow[t]{2}{*}{$P$ value } \\
\hline & SCZ cases & $\mathrm{HC}$ & & \\
\hline A-G-Ins & 41.6 & 37.86 & 1 & - \\
\hline A-C-Ins & 40.82 & 28.09 & $1.28(0.86-1.91)$ & 0.23 \\
\hline G-G-Ins & 2.48 & 12.96 & $0.19(0.06-0.59)$ & $0.0038^{*}$ \\
\hline A-C-Del & 7.91 & 7.38 & $1.00(0.49-2.03)$ & 1 \\
\hline
\end{tabular}

${ }^{*} P<0.05$.

of schizophrenia (SCZ). In line with it, chemokine genes have been candidates for the etiology of this disorder based on their role in driving inflammation and immune responses and regulating many neuronal functions [28]. 
Our results showed that three polymorphisms, regulating positively the expression of the CCL2 chemokine, could have a protective effect on the schizophrenia susceptibility. Indeed, the higher prevalence of the MCP-1 $-2518 \mathrm{G}$ allele in controls as compared to SCZ patients in the current study suggests that the minor $G$ allele acts as a protective factor against SCZ. Our findings go in the same direction as those reported in the Korean population showing that the $\mathrm{G}$ allele frequency is significantly lower in major depressive disorder [19] and tends toward significance in schizophrenia with positive symptomatology [29]. But the contradiction in the previous reports indicated no significant association between any allele and SCZ in Italian [30] and Turkish [31] populations nor the recent study in an Armenian sample [32] which found that the G allele dominantly conferred disease susceptibility.

First, we verified that the genetic frequencies identified in the controls of our study were similar to those reported in other Tunisian studies analyzing different pathologies, which eliminates the possibility of handling errors [33-35]. Also, the discrepancies cited above cannot be only explained by diverse racial and ethnic origins. We noted that the genetic frequencies of controls that were found in our study are similar to those shown in Italian [30], Turkish [31], and Armenian [32] population but differed a lot from those described in the Korean population [19, 29]. The disparity in the results might rather be explained by the inclusion criteria, that is, small sample size and the individual-level factors including lifestyle, dietary variations, and environmental variables. For instance, cases consisted of one hundred and three and they were all with paranoid schizophrenia in the Armenian study [32]. This clinical former represents only $32 \%$ of patients in our work. Concerning the Italian study [30], 35 out of 191 subjects met the criteria for schizoaffective and catatonic disorders, which were excluded in our selection.

For MCP1 -2581G, several studies have demonstrated increased gene expression in vitro and elevated MCP-1 plasma levels in vivo [18, 36-38]. Moreover, a Tunisian study reported that CCL2 plasma concentrations were higher in both asthmatic patients and controls carrying the G allele than in subjects with A polymorphism [39], and these suggest that the $G$ allele could correlate with a higher level of MCP-1 in our Tunisian general population.

We also report, for the first time, a similar genetic correlation between the proximal -362 G/C SNP and schizophrenia where the frequency of the wild $G$ allele is higher in the controls compared to the patients suggesting its protecting effect. Results of the reporter gene assay showed that both variants, MCP1 $-362 \mathrm{G}$ and Ins554-567, exert a higher transcriptional activity, eventually resulting in higher production of MCP-1 [40]. It can be suggested that the $-362 \mathrm{G}$ allele could induce a protective effect against schizophrenia via a production of higher level of MCP-1. In connection with these previous results, the haplotypic analysis showed that the -2581G/-362G/INS haplotype carrying the two alleles associated with higher production of MCP-1 conferred the highest significant difference $(\mathrm{OR}=0.19$, CI $0.06-0.59, P=0.0038)$ compared to the wild- type combination. Haplotype distribution profile suggests that the observed protection status may be driven more strongly by the MCP-1 $-2518 \mathrm{G}$ allele with $-362 \mathrm{G}$ variants, whereas the effect of int1del554-567 may in part be explained by its LD with -362 .

As far as we know, this is the first report of these three variants being examined for association with SCZ. This correlation persisted after adjusting data for confounding factors using logistic regression. The pleiotropic actions of this chemokine on the CNS could explain its involvement in the pathophysiology of schizophrenia. First, the beneficial property of CCL2 in the brain could result from its known role in Th2 polarization. Indeed, higher concentrations of MCP-1 can suppress IFN- $\gamma$ [41] and IL-12p40 production, a common component of IL12 and IL23 [36, 42], and upregulate IL4, the differentiation of Th0 cells into Th2 cells in the peripheral lymphoid organs. It results in a Th2 immunological reaction predominance and underactive the Th1 and/or Th17 immunological reaction [43]. These findings support the Th1/Th2 immune system imbalance hypothesis in schizophrenia, suggesting that Th1 response increases the schizophrenia susceptibility $[2,44]$.

Second, it was shown that CCL2 has the capacity to modulate the release of some brain neurotransmitters. It was revealed that CCL2 enhances AMPA and NMDA receptor currents in spinal neurons and modulates via a presynaptic effect glutamate secretion [45]. In addition, a strong decrease of the transcripts related to glutamatergic neurotransmission and dysregulation of glutamatergic pathways in schizophrenic patients were provided by using DNA microarray techniques [46, 47] and functional neuroimaging studies $[48,49]$.

Third, the extensive and dynamic expression of CCL2 during in utero development suggests a crucial role of this chemokine in the neurogenesis process as promoting the growth of dendrites and synapses [50]. Specifically, numerous chemokines including CCL2 have been involved in the regulation of neural stem/progenitor cell migration, proliferation, differentiation, and integration of new neurons into functional circuits [51]. Implicitly, altered expression in this chemokine network could induce a disruption of early neurodevelopment which has been implicated in many psychiatric disorders, particularly schizophrenia where a number of prenatal maternal environmental factors have been proposed as risk factors for the development of this disorder [52-54]. The levels of MCP-1 were investigated in schizophrenia. Findings are equivocal with nearly equal numbers of studies showing significantly higher $[8,55,56]$ or similar $[57,58]$ levels between schizophrenia patients and healthy comparison subjects (HCs) probably linked to the heterogeneity of clinical features of patients. These results and the high-lighted correlation of MCP-1 genetic polymorphism with positive symptoms scale strengthen the involvement of MCP-1 in the schizophrenia development.

In conclusion, our results identified the haplotype combination -2581G/-362G/int1del554-567 that could mediate protection against schizophrenia in the Tunisian population. These findings are to be extended by additional 
studies, including the monitoring of the MCP-1 protein levels in longitudinal studies, which can help to assess the functional significance of the observed preventing genotypes and haplotypes to schizophrenia.

\section{Data Availability}

No dataset were used to support this study.

\section{Disclosure}

The authors alone are responsible for the content and writing of the paper.

\section{Conflicts of Interest}

The authors declare that they have no conflicts of interest.

\section{Acknowledgments}

We are deeply grateful to all patients and healthy controls that participated in this study. We would also like to acknowledge the staff of the Department of Psychiatry of the CHU F. Bourguiba of Monastir and the crew of blood bank in the same hospital for providing samples and clinical information. This work was supported by grants from the Ministry of Higher Education, Scientific Research and Technology of Tunisia.

\section{References}

[1] D. A. Lewis and J. A. Lieberman, "Catching up on schizophrenia,” Neuron, vol. 28, no. 2, pp. 325-334, 2000.

[2] B. J. Miller, P. Buckley, W. Seabolt, A. Mellor, and B. Kirkpatrick, "Meta-analysis of cytokine alterations in schizophrenia: clinical status and antipsychotic effects," Biological Psychiatry, vol. 70, no. 7, pp. 663-671, 2011.

[3] A. Bergon, R. Belzeaux, M. Comte et al., "CX3CR1 is dysregulated in blood and brain from schizophrenia patients," Schizophrenia Research, vol. 168, no. 1-2, pp. 434-443, 2015.

[4] S. Najjar, D. M. Pearlman, K. Alper, A. Najjar, and O. Devinsky, "Neuroinflammation and psychiatric illness," Neuroinflammation, vol. 10, pp. 43-67, 2013.

[5] A. Sekar, A. R. Bialas, H. de Rivera et al., "Schizophrenia risk from complex variation of complement component 4," $\mathrm{Na}$ ture, vol. 530, no. 7589, pp. 177-183, 2016.

[6] H. Kettenmann, U.-K. Hanisch, M. Noda, and A. Verkhratsky, "Physiology of microglia," Physiological Reviews, vol. 91, no. 2, pp. 461-553, 2011.

[7] K. Saijo and C. K. Glass, "Microglial cell origin and phenotypes in health and disease," Nature Reviews Immunology, vol. 11, no. 11, pp. 775-787, 2011.

[8] D. Frydecka, M. Krzystek-Korpacka, A. Lubeiro et al., "Profiling inflammatory signatures of schizophrenia: a crosssectional and meta- analysis study," Brain, Behavior, and Immunity, vol. 71, pp. 28-36, 2018.

[9] F. Orhan, L. Schwieler, H. Fatouros-Bergman et al., "Increased number of monocytes and plasma levels of MCP-1 and YKL40 in first-episode psychosis," Acta Psychiatrica Scandinavica, vol. 138, no. 5, pp. 432-440, 2018.

[10] A. R. Glabinski, V. Balasingam, M. Tani, S. L. Kunkel, R. M. Strieter, and V. W. Yong, "Chemokine monocyte chemoattractant protein-1 is expressed by astrocytes after mechanical injury to the brain," Journal of Immunology, vol. 156, no. 11, pp. 4363-4368, 1996.

[11] U.-K. Hanisch, "Microglia as a source and target of cytokines," Glia, vol. 40, no. 2, pp. 140-155, 2002.

[12] B. P. Barna, J. Pettay, G. H. Barnett, P. Zhou, K. Iwasaki, and M. L. Estes, "Regulation of monocyte chemoattractant protein-1 expression in adult human non-neoplastic astrocytes is sensitive to tumor necrosis factor (TNF) or antibody to the 55$\mathrm{kDa}$ TNF receptor," Journal of Neuroimmunology, vol. 50, no. 1, pp. 101-107, 1994.

[13] J. W. Berman, M. P. Guida, J. Warren, J. Amat, and C. F. Brosnan, "Localization of monocyte chemoattractant peptide-1 expression in the central nervous system in experimental autoimmune encephalomyelitis and trauma in the rat," Journal of Immunology, vol. 156, pp. 3017-3023, 1996, https://www.jimmunol.org/content/156/8/3017.short.

[14] G. Banisadr, R.-D. Gosselin, P. Mechighel, P. Kitabgi, W. Rostène, and S. M. Parsadaniantz, "Highly regionalized neuronal expression of monocyte chemoattractant protein-1 (MCP-1/CCL2) in rat brain: evidence for its colocalization with neurotransmitters and neuropeptides," The Journal of Comparative Neurology, vol. 489, no. 3, pp. 275-292, 2005.

[15] M. Hayashi, Y. Luo, J. Laning, R. M. Strieter, and M. E. Dorf, "Production and function of monocyte chemoattractant protein- 1 and other $\beta$-chemokines in murine glial cells," Journal of Neuroimmunology, vol. 60, no. 1-2, pp. 143-150, 1995.

[16] L. S. Da, Y. Zhang, S. Zhang et al., "Association between MCP-1 -2518A/G polymorphism and cancer risk: evidence from 19 case-control studies," PLoS One, vol. 8, Article ID e82855, 2013.

[17] P. Nyquist, J. Zhang, and T. J. De Graba, “The -928 G/C and -362 G/C single-nucleotide Polymorphisms in the Promoter of MCP-1: increased transcriptional activity and novel binding sites," Cerebrovascular Diseases, vol. 29, no. 3, pp. 242-247, 2010.

[18] B. H. Rovin, L. Lu, and R. Saxena, "A novel polymorphism in the MCP-1 gene regulatory region that influences MCP-1 expression," Biochemical and Biophysical Research Communications, vol. 259, no. 2, pp. 344-348, 1999.

[19] C.-U. Pae, H.-S. Yu, T.-S. Kim et al., "Monocyte chemoattractant protein-1 (MCP1) promoter -2518 polymorphism may confer a susceptibility to major depressive disorder in the Korean population," Psychiatry Research, vol. 127, no. 3, pp. 279-281, 2004.

[20] J. Losy, G. Michałowska-Wender, A. Kurdyn'ska, and M. Wender, "CCL2 (MCP-1) and CCL5 (RANTES) levels in the peripheral blood of multiple sclerosis patients treated with Glatiramer Acetate (Copaxone)," Folia Neuropathologica, vol. 43, pp. 153-155, 2005.

[21] R. Pola, A. Flex, E. Gaetani et al., "Monocyte chemoattractant protein-1 (MCP-1) gene polymorphism and risk of Alzheimer's disease in Italians," Experimental Gerontology, vol. 39, no. 8, pp. 1249-1252, 2004.

[22] C. Guo, H. Zhang, Q. Gao et al., "Monocyte chemoattractant protein-1 in spinal tuberculosis: -362G/C genetic variant and protein levels in Chinese patients," Diagnostic Microbiology and Infectious Disease, vol. 78, no. 1, pp. 49-52, 2014.

[23] H. Chinoy, F. Salway, N. Fertig et al., "Monocyte chemotactic protein-1 single nucleotide polymorphisms do not confer susceptibility for the development of adult onset polymyositis/dermatomyositis in UK Caucasians," Rheumatology, vol. 46, no. 4, pp. 604-607, 2006.

[24] American Psychiatric Association, Diagnostic and Statistical Manual of Mental Disorders, American Psychiatric Association, Washington, DC, USA, Fourth Edition, 1994. 
[25] H. Y. Meltzer, J. Rabinowitz, M. A. Lee et al., "Age at onset and gender of schizophrenic patients in relation to neuroleptic resistance," American Journal of Psychiatry, vol. 15, no. 4, pp. 475-482, 1997.

[26] A. S. Al-Ansari, W. E. R. Ollier, J. Villarreal, J. Ordi, L.-S. Teh, and A. H. Hajeer, "Tumor necrosis factor receptor II (TNFRII) exon 6 polymorphism in systemic lupus erythematosus," Tissue Antigens, vol. 55, no. 1, pp. 97-99, 2000.

[27] J. C. Barrett, B. Fry, J. Maller, and M. J. Daly, "Haploview: analysis and visualization of LD and haplotype maps," Bioinformatics, vol. 21, no. 2, pp. 263-265, 2004.

[28] M. J. Stuart, G. Singhal, and B. T. Baune, "Systematic review of the neurobiological relevance of chemokines to Psychiatric disorders," Frontiers in Cellular Neuroscience, vol. 9, pp. 357-372, 2015.

[29] C.-U. Pae, K.-I. Chung, J.-J. Kim et al., "Monocyte chemoattractant protein-1 promoter -2518 polymorphism and schizophrenia in the Korean population," Psychiatric Genetics, vol. 14, no. 2, pp. 65-67, 2004.

[30] E. Mundo, A. C. Altamura, S. Vismara et al., "MCP-1 gene (SCYA2) and schizophrenia: a case-control association study," American Journal of Medical Genetics Part B: Neuropsychiatric Genetics, vol. 132, no. 1, pp. 1-4, 2005.

[31] S. Dasdemir, C. I. Kucukali, E. S. Bireller, E. Tuzun, and B. Cakmakoglu, "Chemokine gene variants in schizophrenia," Nordic Journal of Psychiatry, vol. 70, no. 6, pp. 407-412, 2016.

[32] Z. Roksana, B. Anna, A. Arsen, M. Maya, M. Frantisek, and P. Martin, "Monocyte chemoattractant protein-1 in schizophrenia: $-2518 \mathrm{~A} / \mathrm{G}$ genetic variant and protein levels in Armenian population,” Cytokine, vol. 58, pp. 351-354, 2012.

[33] R. Jemaa, S. Ben Ali, A. Kallel et al., "Association between the -2518G/A polymorphism in the monocyte chemoattractant protein-1 (MCP-1) gene and hypertension in Tunisian patients," Clinical Biochimistry, vol. 42, pp. 34-37, 2008.

[34] W. Ben-Selma, H. Harizi, and J. Boukadida, "MCP-1 -2518 A/ $\mathrm{G}$ functional polymorphism is associated with increased susceptibility to active pulmonary tuberculosis in Tunisian patients," Molecular Biology Reports, vol. 38, no. 8, pp. 5413-5419, 2011.

[35] A. Messadi, N. Fekih-Mrissa, A. Kallel et al., "Lack of association between monocyte protein-1 (MCP-1) -2518 A>G chemoattractant and $\mathrm{C}-\mathrm{C}$ chemokine receptor 2 (CCR2) Val64Ile polymorphisms and multiple sclerosis in a Tunisian population," Journal of Clinical Neuroscience, vol. 17, no. 10, pp. 1311-1313, 2010.

[36] P. O. Flores-Villanueva, J. A. Ruiz-Morales, C.-H. Song et al., "A functional promoter polymorphism inmonocyte chemoattractant protein-1is associated with increased susceptibility to pulmonary tuberculosis," The Journal of Experimental Medicine, vol. 202, no. 12, pp. 1649-1658, 2005.

[37] M. Ganachari, J. A. Ruiz-Morales, J. C. Pretell, J. Dinh, J. Granados, and P. O. Flores-Villanueva, "Joint effect of MCP-1 genotype GG and MMP-1 genotype 2G/2G increases the likelihood of developing pulmonary tuberculosis in BCGvaccinated individuals," PLoS One, vol. 5, Article ID e8881, 2010 .

[38] K. S. Brown, E. Nackos, S. Morthala, L. E. Jensen, A. S. Whitehead, and J. M. Von Feldt, "Monocyte chemoattractant protein-1: plasma concentrations and A(-2518)G promoter polymorphism of its gene in systemic lupus erythematosus," Rheumatology, vol. 34, pp. 740-746, 2007, http://citeseerx.ist.psu.edu/viewdoc/ download?doi=10.1.1.956.5338\&rep=rep1\&type=pdf.

[39] H. Chelbi, A. Ghadiri, J. Lacheb et al., "A polymorphism in the CCL2 chemokine gene is associated with asthma risk: a case- control and a family study in Tunisia," Genes \& Immunity, vol. 9, no. 7, pp. 575-581, 2008.

[40] C. D. Intemann, T. Thye, B. Förster et al., "MCP1 haplotypes associated with protection from pulmonary tuberculosis," BMC Genetics, vol. 12, no. 1, pp. 34-43, 2011.

[41] I. F. Charo, R. M. Ransohoff, and R. M., "The many roles of chemokines and chemokine receptors in inflammation," New England Journal of Medicine, vol. 354, no. 6, pp. 610-621, 2006.

[42] G. Trinchieri, "Interleukin-12 and the regulation of innate resistance and adaptive immunity," Nature Reviews Immunology, vol. 3, no. 2, pp. 133-146, 2003.

[43] A. Mendez, R. Hernandez-Pando, S. Contreras, D. Aguilar, and G. A. W. Rook, "CCL2, CCL18 and sIL-4R in renal, meningeal and pulmonary TB; a 2 year study of patients and contacts," Tuberculosis, vol. 91, no. 2, pp. 140-145, 2011.

[44] L. Srinivas, N. N. Vellichirammal, A. M. Alex et al., "Proinflammatory cytokines and their epistatic interactions in genetic susceptibility to schizophrenia," Neuroinflammation, vol. 13, no. 1, pp. 105-117, 2016.

[45] Y.-J. Gao, L. Zhang, O. A. Samad et al., "JNK-induced MCP-1 production in spinal cord astrocytes contributes to central sensitization and neuropathic pain," Journal of Neuroscience, vol. 29, no. 13, pp. 4096-4108, 2009.

[46] K. Mirnics, F. A. Middleton, A. Marquez, D. A. Lewis, and P. Levitt, "Molecular characterization of schizophrenia viewed by microarray analysis of gene expression in prefrontal cortex," Neuron, vol. 28, no. 1, pp. 53-67, 2000.

[47] W. G. Frankle, J. Lerma, and M. Laruelle, "The synaptic hypothesis of schizophrenia," Neuron, vol. 39, no. 2, pp. 205-216, 2003.

[48] A. Gozzi, C. H. Large, A. Schwarz, S. Bertani, V. Crestan, and A. Bifone, "Differential effects of antipsychotic and glutamatergic agents on the phMRI response to phencyclidine," Neuropsychopharmacology, vol. 33, no. 7, pp. 1690-1703, 2008.

[49] L. T. van Elst, G. Valerius, M. Buchert et al., "Increased prefrontal and hippocampal glutamate concentration in schizophrenia: evidence from a magnetic resonance spectroscopy study," Biological Psychiatry, vol. 58, no. 9, pp. 724-730, 2005.

[50] S. Z. Meng, A. Oka, and S. Takashima, "Developmental expression of monocyte chemoattractant protein-1 in the human cerebellum and brain stem," Brain and Development, vol. 21, no. 1, pp. 30-35, 1999.

[51] S. Chintawar, R. Cayrol, J. Antel, M. Pandolfo, and A. Prat, "Blood-brain barrier promotes differentiation of human fetal neural precursor cells," Stem Cells, vol. 27, no. 4, pp. 838-846, 2009.

[52] S. K. Hunter, M. A. Kisley, L. McCarthy, R. Freedman, and R. G. Ross, "Diminished cerebral inhibition in neonates associated with risk factors for schizophrenia: parental psychosis, maternal depression, and nicotine use," Schizophrenia Bulletin, vol. 37, no. 6, pp. 1200-1208, 2011.

[53] K. S. Betts, G. M. Williams, J. M. Najman, J. Scott, and R. Alati, "Exposure to stressful life events during pregnancy predicts psychotic experiences via behaviour problems in childhood," Journal of Psychiatric Research, vol. 59, pp. 132-139, 2014.

[54] K. S. Betts, G. M. Williams, J. M. Najman, J. Scott, and R. Alati, "Maternal prenatal infection, early susceptibility to illness and adult psychotic experiences: a birth cohort study," Schizophrenia Research, vol. 156, no. 2-3, pp. 161-167, 2014.

[55] W. Beumer, R. C. Drexhage, H. De Wit et al., "Increased level of serum cytokines, chemokines and adipokines in patients 
with schizophrenia is associated with disease and metabolic syndrome," Psychoneuroendocrinology, vol. 37, no. 12, pp. 1901-1911, 2012.

[56] D. H. Dimitrov, S. Lee, J. Yantis et al., "Differential correlations between inflammatory cytokines and psychopathology in veterans with schizophrenia: potential role for IL-17 pathway," Schizophrenia Research, vol. 151, no. 1-3, pp. 29-35, 2013.

[57] P. Brambilla, M. Bellani, M. Isola et al., "Increased M1/decreased M2 signature and signs of Th1/Th2 shift in chronic patients with bipolar disorder, but not in those with schizophrenia," Translational Psychiatry, vol. 4, pp. 406-413, 2014.

[58] M. Di Nicola, A. Cattaneo, N. Hepgul et al., "Serum and gene expression profile of cytokines in first-episode psychosis," Brain, Behavior, and Immunity, vol. 31, pp. 90-95, 2013. 


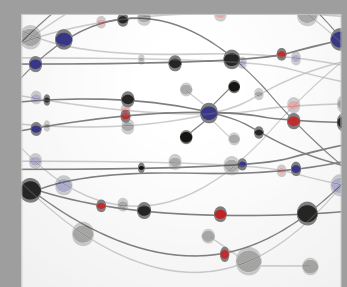

The Scientific World Journal
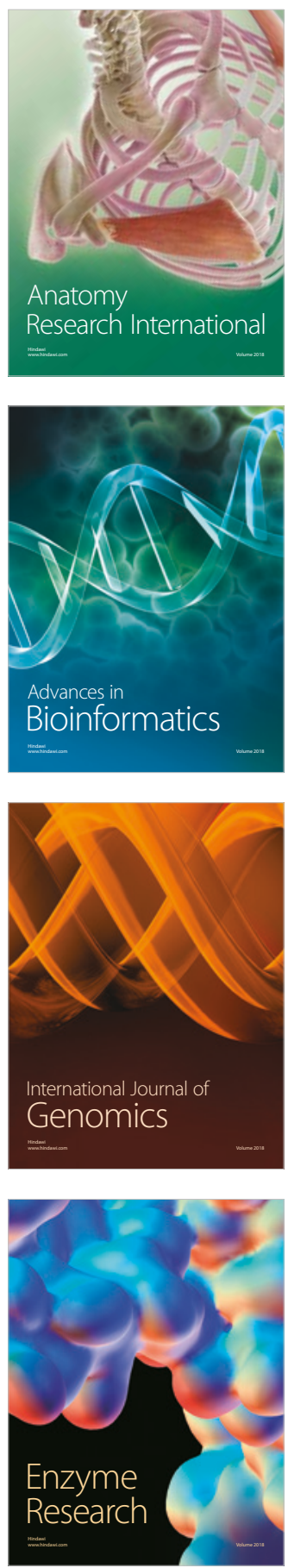
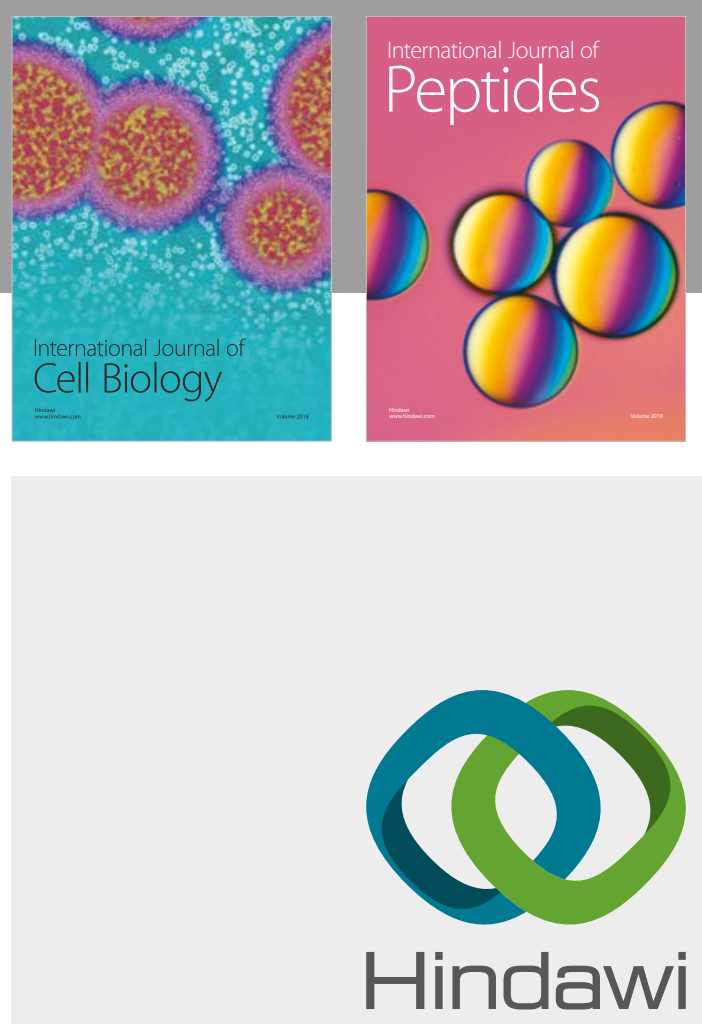

Submit your manuscripts at

www.hindawi.com
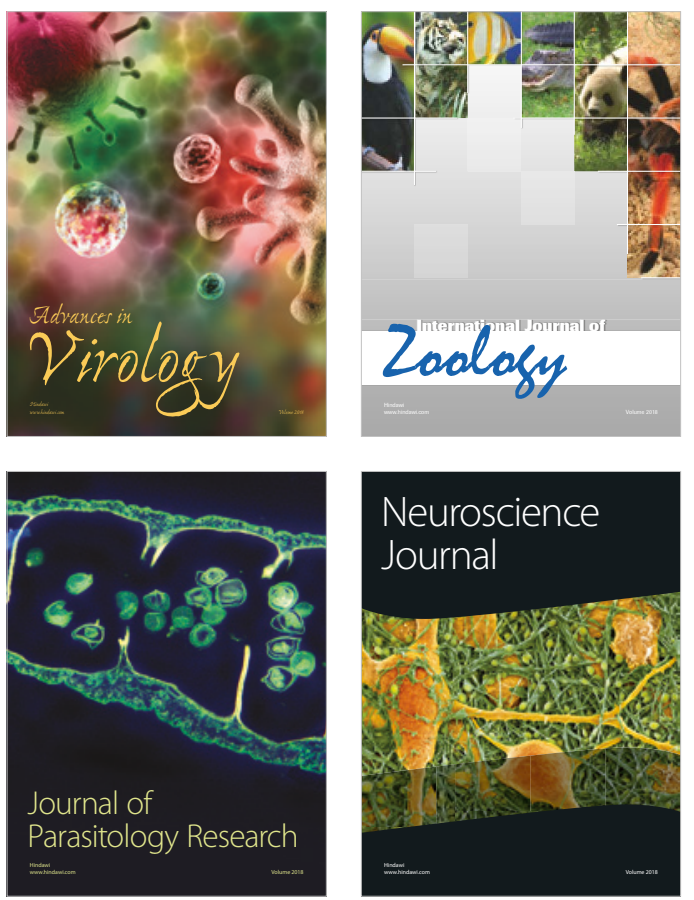
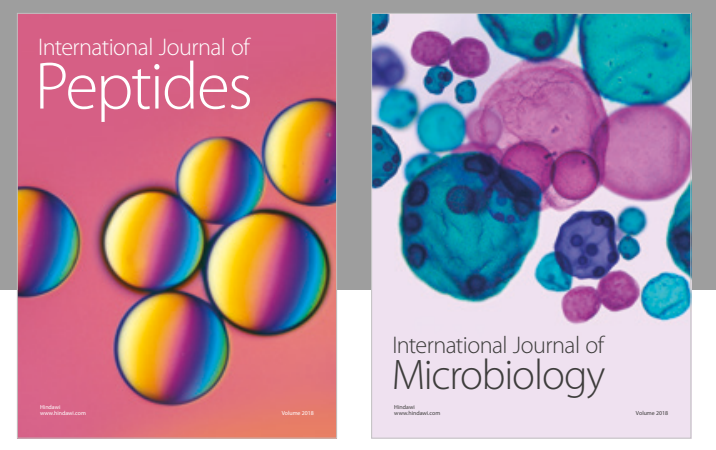

nternational Journal of Microbiology
Journal of
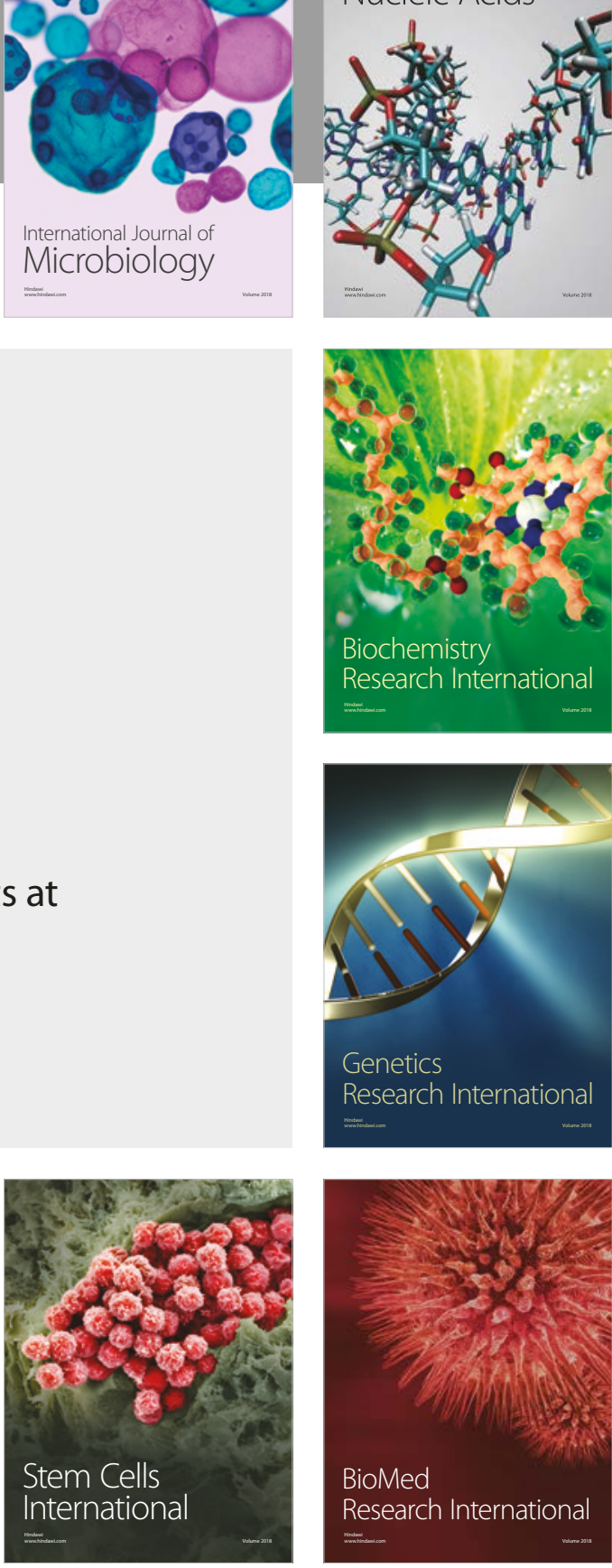
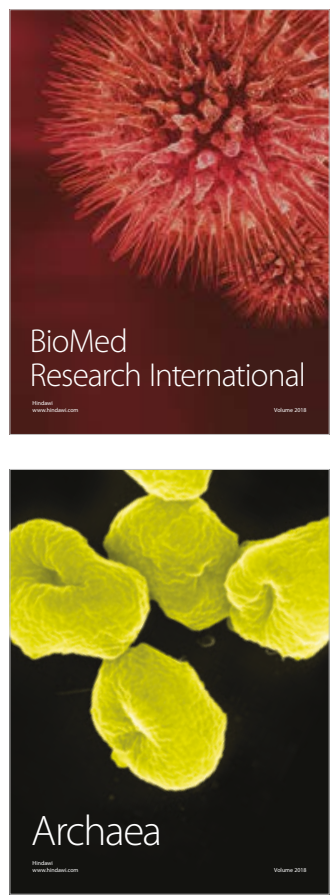